\title{
A COLOUR HISTOGRAM BASED APPROACH TO HUMAN FACE DETECTION
}

\author{
Jianzhong Fang and Guoping Qiu
}

\author{
School of Computer Science, The University of Nottingham
}

\begin{abstract}
In this paper, we introduce a novel colour histogram based approach to human face detection in colour images. We reason that different regions of a human face contain different facial features and that the colour distributions of these regions may have certain characteristics that are unique to human faces. By concatenating colour histograms of different regions of a human face, we can form a vector that captures the spatial relationship of these unique regional characteristics, which in turn enables the development of effective face detection schemes. To improve efficiency and detection speed, we use principal component analysis (PCA) to reduce the dimensionality of the histograms and apply skin detection as a pre-processing step to reduce the search space. We use support vector machine for both skin detection and face detection. We present experimental results to demonstrate the effectiveness of the new method
\end{abstract}

\section{INTRODUCTION}

Face detection is a well-known pattern recognition problem. Although many approaches, from feature-based to appearancebased, have been proposed over the years, it still remains a very challenging problem today $[1,2]$.

Earlier face detection methods are mostly feature-based, and newer methods are mostly appearance-based. Feature-based approaches detect faces by first detecting distinct components of the face, such as the eyes, the nose and the mouth and then by measuring the geometric relations of these features. The main difficulty of these approaches is that it is very difficult to translate human knowledge (about the face) to computer representation, and reliable facial feature detection is still a research problem. Appearance-based approaches do not require the detection of specific facial features, and build detection models directly from the image data, often via some form of learning techniques [3 - 7].

This paper proposes a new appearance-based approach to face detection in colour images. The schematic of the new method is illustrated in Fig.1. In this new method, a test subimage is first normalised to a fixed size (64 x 80 pixels is used in our current work). Colour histograms of overlapping sub regions of the test image are then compiled. These colour histograms are then compressed by the Karhunen-Loeve Transform (KLT) to a set of compact representation coefficients which are then presented to a support vector machine (SVM) [11] to detect the presence/absence of human face in the test sub image. To reduce searching time, SVM-based skin detection is also implemented to identify potential facial locations thus reducing the search area and enhancing the detection speed.

\section{FACE REPRESENTATION USING MULTIPLE COLOUR HISTOGRAMS}

RGB colour space is commonly used in image processing because of its basic synthesis property and direct application in image display. According to the requirements of different image processing tasks, RGB colour space is often transformed to other colour spaces. From a visual perception's point of view, hue, saturation and value are often employed to manipulate colour, such as de-saturation or change of colourfulness. When the colour is quantized to a limit number of representative colours, one will have to deal with two problems. The first is how to best match the distance of data representation to human perception. It is desirable that numerical colour distance is proportional to perceptual difference. The second problem is how to best quantize the colours such that the reproductions from these quantized colours is the most faithful to the original. In this work, we adopt a perceptually meaningful colour space, the HMMD colour space, and used a carefully worked out quantization scheme of the MPEG-7 standard [10].

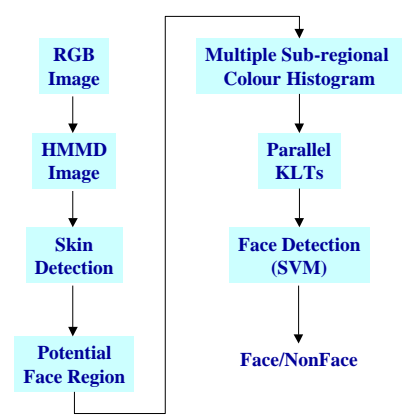

Fig. 1 The schematic of the new face detection method

\subsection{Face Representation based on Multiple Colour Histograms}

Colour histogram is a global statistical measure of an image. A global colour histogram will have very limited discriminative power. Researchers have developed various lowlevel features for face detection. Some techniques use raw pixels to represent a face image by a long vector of pixel values and put all the decision burdens on the decision (learning) module [5]. Other techniques divide a face image into sub-regions and use KLT to compress the raw pixels to a lower dimensional vector before sending it to the decision module [3]. Yet other techniques use some form of transform (Wavelet) to extract lowlevel features for further processing and detection [6].

Face images have their unique characteristics. In order to develop effective techniques that can distinguish face images 
from non-face images, it is necessary to exploit the uniqueness of face. The first feature that can be exploited is that a face is mostly covered by skin colour and skin colour can be detected to a very high accuracy. Skin colour based methods have been used extensively for face detection $[8,9]$. Therefore we believe colour histogram could be a useful cue for face detection. However, colour histogram alone will not be very useful for the obvious reasons, i.e., there may be other skin colour objects in the image that are not faces. However, if we divide a face image into subregions, as shown in Fig. 2, each region covers various facial features, then the colour distribution of each region may have features that are unique to face images. Therefore the histograms of the sub regions may have their unique characteristics, and a combination of these histograms may be used to form discriminative feature vectors for effective face detection. It is based on this reasoning we introduce a multiple colour histogram based face representation scheme. Let HR(i) be the colour histogram of face region $i$, then a face (or non face) is represented by a feature vector $\mathrm{H}$ as

$$
\mathrm{H}=\{\operatorname{HR}(1), \operatorname{HR}(2), \ldots, \operatorname{HR}(\mathrm{M})\}
$$

We believe with such a representation scheme that $\mathrm{p}(\mathrm{H} \mid$ Face $)$ should be very different from $\mathrm{p}(\mathrm{H} \mid$ Non-Face $)$, thus enabling effective face detection. However, to use (1) as a feature, there are a number of practical issues, and we address two of them. Firstly, in order to be reasonably accurate, the number of colour histogram bins should be relative large, and a combination of multiple histograms will likely to result in a $\mathrm{H}$ that is of very high dimensions. High dimensionality will have the "curse of dimensionality" problem as well as practical computational problems. Secondly, there is the issue of how to build the classifier using the feature of (1). In this paper, we use principal component analysis to reduce the dimensionality of the histogram and we use support vector machine to classify whether a vector $\mathrm{H}$ belongs to face or non-face.

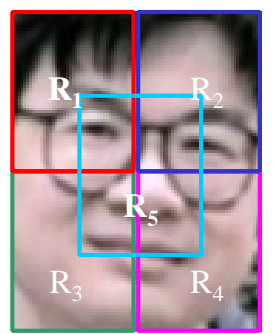

Fig.2 A face image may be divided into multiple sub regions and each region may have unique colour distributions.

\subsection{Dimensionality Reduction via Karhunen-Loeve Transform}

The features represented by (1) will be of very high dimension. One of the popular techniques that is used for dimensionality is the principal component analysis (PCA) or equivalently Karhunen-Loeve Transform (KLT). This transform has been used extensively in machine vision and pattern recognition, and it is also popular in face detection [1,2]. There are a number of possibilities to implement PCA to compress features derived from (1). One possibility is to view $\mathrm{H}$ as a single vector and project $\mathrm{H}$ onto its eigen space. However, since $\mathrm{H}$ is of high dimension, the co-variance matrix could be prohibitory large from a computational point of view. On the other hand, the histogram of a sub region may be of a more manageable dimension. Therefore, instead of compressing $\mathrm{H}$ as a single vector, we compress the regional histogram, HR(i), separately, and then concatenate the projections to form a compressed feature vector. Let $\mathrm{HR}(\mathrm{i})=\left\{\mathrm{hr}_{1}(\mathrm{i}), \mathrm{hr}_{2}(\mathrm{i}), \ldots, \mathrm{hr}_{\mathrm{N}}(\mathrm{i})\right\}$ be the colour histogram of region $\mathrm{i},\left\{\Phi_{\mathrm{k}}(\mathrm{i}), \mathrm{k}=1,2, \ldots \mathrm{N}\right\}$ be the $\mathrm{k}$-th eigen vector of the ith region, then, $\operatorname{PHR}(\mathrm{i})=\left\{\mathrm{phr}_{1}(\mathrm{i}), \mathrm{phr}_{2}(\mathrm{i})\right.$, $\left.\ldots, \operatorname{phr}_{\mathrm{N}}(\mathrm{i})\right\}$ the projections of HR(i) in the eigen space, then we have

$$
\operatorname{phr}_{\mathrm{k}}(\mathrm{i})=\Phi_{\mathrm{k}}(\mathrm{i}) \mathrm{HR}(\mathrm{i})
$$

It is well known that after the transform, most of the energy of PHR(i) will be concentrated in the first few coefficients, we can therefore approximate the original histogram HR(i) by a much shorter vector HR'(i) as

$\operatorname{HR}^{\prime}(\mathrm{i})=\left\{\operatorname{phr}_{1}(\mathrm{i}), \operatorname{phr}_{2}(\mathrm{i}), \ldots, \operatorname{phr}_{\mathrm{n}}(\mathrm{i})\right\}$, where $\mathrm{n}<\mathrm{N}$

To choose an appropriate $n$, we compute the following energy preservation criterion

$$
E=\sum_{k \leq n} \lambda_{k} / \sum_{\forall k} \lambda_{k}
$$

where $\lambda_{\mathrm{k}}$ is the k-th eigen value of the co-variance matrix. We found that on average, by setting $\mathrm{E}=90 \%$, significant compression (>15:1) can be achieved without affecting detection performance. After transform, the following feature now approximates the feature in (1)

$$
\mathrm{H}^{\prime}=\left\{\mathrm{HR}^{\prime}(1), \mathrm{HR}^{\prime}(2), \ldots, \mathrm{HR}^{\prime}(\mathrm{M})\right\}
$$

A face or non-face image is now represented by KLT compressed multiple colour histograms (5). This representation can then be used to construct detection model.

\section{FACE DETECTION STRATEGY}

In this work, we adopt an exhaustive search approach that is used often by researchers to detect possible face in an image [3]. In this scheme, image windows of various predefined sizes are moved across the image. When a window is moved to a new position, a decision is made whether a face is contained inside the window. It is not difficult to see that such a scheme will be computationally very expensive because it has to search all locations of the image for all possible window sizes. In order to reduce the search effort, in this work, we first perform skin detection using a support vector machine based approach. Then face search will be only performed in those areas that contain skin colour. Using such a scheme, we can increase the detection speed dramatically.

\subsection{Skin Detection}

Skin colour distribution has been shown to have its unique characteristics. There have been a number of face detection techniques that are based on the detection of skin colours, e.g., [8]. In this work, we have developed a SVM based method to detection skin. In this scheme, we divide the image into overlapping $4 \times 4$ blocks and process one block at a time, each time determining whether the center $2 \times 2$ pixels of the block are 
skin pixels. From the $4 \times 4$ colour block, we derive a 14 dimensional vector representing the colour and texture properties of the block. This 14-d vector is then presented to a SVM which are trained to make a binary decision, i.e., the center $2 \times 2$ pixels of the block belong to skin/non-skin. An example of skin detection based on this method is shown in Fig. 3. We have found that this method is very efficient and very effective in detecting skin regions.

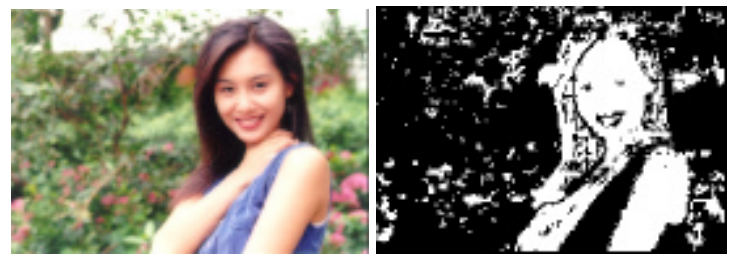

Fig.3 Left: Original image Right: Skin (white colour)

\subsection{Support Vector Machine for Face Detection}

For a potential face region, we need to have a model which will use the representation features vector of (5) as input and decide whether the region is a face. In the literature, neural network has been demonstrated to give impressive performance [4], statistical decision models [3] have also been shown to be very effective. One of the machine learning techniques that has emerged as a promising tools for pattern recognition is Support Vector Machine (SVM) [11]. It has been successfully used in face detection [7]. In this work, we will build our decision module using SVM.

\section{EXPERIMENTAL RESULTS}

Our face database consists of face images of undergraduate students in our department. These images were taken by a lowend digital camera when the students first entered the department. To create a training database, the original face samples with various sizes are normalised to the size of 64 × 80 (width $\mathrm{x}$ height) pixels. There are about 500 original faces in the training set. In order to generate more training samples, these faces are rotated up to 14 degrees in both directions, to generate a total of 2083 training face samples. We collect non-face samples of size $64 \times 80$ pixels from various categories of the Corel data set. The categories of images we use to collect non face samples include scenery, texture, surface, fur, desert, underwater shimmer, art and skin patches, and there are 3000 such patches in the training set. For each face and non-face sample image, we divide it into five sub regions of size $32 \times 40$ as shown in Fig. 2. For each region, we compile a 128-bin colour histogram in the HMMD space based on the quantization scheme recommended in MPEG-7 standard. The original vector according to (1) therefore is $5 \times 128=640$ dimensional. We use KLT as described in section 2.3 to compress these histograms to obtain a vector according to (5) that is 70 dimensional, achieving a 9 fold compression. Face samples are tagged with +1 , while non-face samples are tagged with -1 , all the face and non-face samples are fed to the SVM for training the machine. We choose RBF kernel and Sequential Minimal Optimisation method to train the SVM. Once the machine is trained, it is ready to be used for face detection.
As mentioned in Section 3.1, we also perform skin detection in order to reduce the amount of search. We train a separate SVM for skin detection using a large number of skin/non-skin samples ( $4 \times 4$ blocks $)$ collected from various sources.

In all tests, we search faces of 14 different sizes, ranging from the smallest of $18 \times 23$ pixels to the biggest of $280 \times 350$ pixels. For each of these 14 sizes, the window is scaled up or down to the standard size of $64 \times 80$ for detection. To speedup the search, only those regions marked as skin by the skin detection SVM are evaluated for possible faces.

In the testing data set, there are 44 images of various colours and textures and in them there are 257 faces of various sizes. Some of the testing images are obtained by embedding those student faces that are outside the training set into images of a variety of colours and texture characteristics, and others were obtained from various sources on the Internet.

A face is counted as correctly detected if the two eyes and the month are within the detection window. If a declared detection window does not include any face feature inside it, then that incident is counted as a false detection. If a declared detection window that covers part of the face but does not have the eyes and mouth fully inside the window then we do not count it as a miss nor as a false detection, we call this situation partial detection. Based on this criterion, detection results are shown in Table 1.

\begin{tabular}{|c|c|c|c|}
\hline $\begin{array}{c}\text { Correct } \\
\text { Detection }\end{array}$ & $\begin{array}{c}\text { Partial } \\
\text { Detection }\end{array}$ & Missed & $\begin{array}{c}\text { False } \\
\text { Detection }\end{array}$ \\
\hline $\mathbf{2 1 5}(\mathbf{8 3 \%})$ & $\mathbf{2 4}(\mathbf{9 \%})$ & $\mathbf{1 8}(\mathbf{7 \%})$ & $\mathbf{2 7}$ \\
\hline
\end{tabular}

Table 1 Face detection results using distributed color histogram and SVM

It is seen that the correct detection rate is high (83\%) and the only $7 \%$ were completely missed. There are 27 false detections, which should be viewed in relation to the number of detection windows $(>1$ million after colour detection) examined by the algorithm. Fig. 4 shows some examples of the detection results. It should be pointed out that the backgrounds of these testing images are very tough with many skin-like color patches. It is seen that the new method works effectively.

In terms of face detection speed, our skin color detection pre-processing has demonstrated very significant advantages. All our simulations were performed on a PC with a Pentium P2 $1.5 \mathrm{GHz}$ processor. For face detection on 256 x 384-pixel images, on average, it took about 30 minutes to process an image using a full search strategy (without skin colour preprocessing). With skin color preprocessing, it took on average just a little under 1.5 minute to process an image. That is, achieving a speed up factor of over 20. (It should be noted in either case the computer source code was not optimized).

\section{CONCLUDING REMARKS}

In this paper, we have introduced a novel colour histogram based technique for face detection. Our rationale is that human face has some unique features and that a good face detection strategy should try to exploit the uniqueness of these features. We argue 
that different regions of a face contain different facial features and that it is likely that the colour distributions of these regions contain unique characteristics. Based on these reasoning, we have developed a multiple colour histogram scheme to represent faces. To improve computational efficiency, we use PCA to reduce the dimensionality of the feature. We have presented experimental results that demonstrate that the new method is very effective in locating human faces in very tough backgrounds. Our current work includes the use of much larger training and testing sets to evaluate the method. We are also investigating fast methods to speed up the technique to enable it to perform real time face tracking in video sequence.

\section{REFERENCES}

1. M-H Yang, D. Kriegman and N. Ahuja, "Detecting Face in Images: A Survey", IEEE Transaction on Pattern Analysis and Machine Intelligence, vol. 24, no. 1, pp. 34-58, January, 2002.

2. E. Hjelmås and B-K Low, "Face Detection: A Survey", Computer Vision and Image Understanding 83, 236-274 (2001).

3. H. Schneiderman and T. Kanade, "Probabilistic Modeling of Local Appearance and Spatial Relationships for Object Recognition",CVPR '98. pp. 45-51.

4. H. Rowley, S. Baluja and T. Kanade, "Neural Network-Based Face Detection", IEEE Transaction on Pattern Analysis and Machine Intelligence, vol. 20, no. 1, pp. 23-38, January 1998

5. K. Sung and T. Poggio, " Example-Based Learning for View-Based Human Face Detection", IEEE Transactions on Pattern Analysis and Machine Intelligence, vol. 20, no. 1, January 1998.

6. Y. Zhu, S. Schwartz and M. Orchard, "Fast Face Detection Using Subspace Discriminant Wavelet Features", CVPR'00.

7. B. Heisele et al, "Component-based Face Detection", CVPR'01.

8. R. Hsu, M. Abdel-Mottaleb and A. K. Jain, " Face Detection in Color Images", IEEE Transaction on Pattern Analysis and Machine Intelligence, vol. 24, no. 5, May 2002-10-08.

9. H. Wu, Q. Chen and M. Yachida, "Face Detection From Color Images Using a Fuzzy Pattern Matching Method", IEEE Transactions on Pattern Analysis and Machine Intelligence, vol. 21, no. 6, JUNE 1999.

10. B. S. Manjunath, et al, " Color and Texture Descriptors", IEEE Transactions on Circuits and Systems for Video Technology, vol. 11, no. 6, JUNE 2001.

11. V. Vapnik, Statistical learning theory, John Wiley and Sons, New York, 1998.

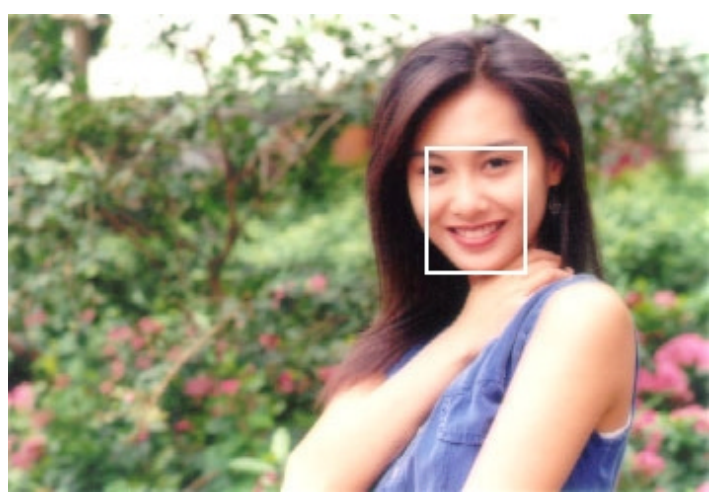

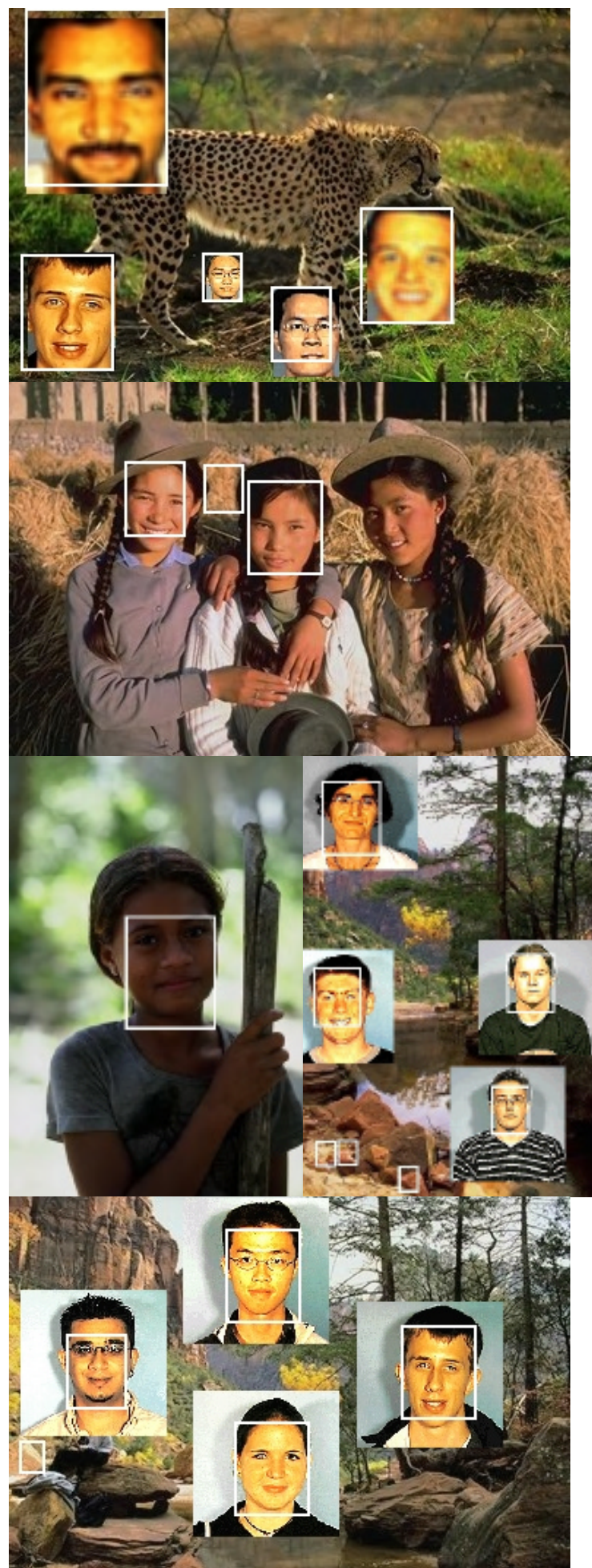

Fig. 4 Examples of testing image and face detection results. Many of our testing images contain skin like colour and surfaces. 